\title{
Phosphorus Status in Soils of Eastern Dry Zone, Karnataka, India
}

\author{
M. Chandrakala ${ }^{1 *}$, C.A. Srinivasamurthy ${ }^{2}$, Sanjeev Kumar ${ }^{3}$, S. Bhaskar ${ }^{4}$, \\ V.R.R. Parama ${ }^{5}$ and D.V. Naveen ${ }^{6}$ \\ ${ }^{1}$ National Bureau of Soil Survey and Land Use Planning, Regional Centre, Hebbal, \\ Bangalore-560 024, Karnataka, India \\ ${ }^{2}$ Director of Research, Central Agricultural University, Imphal, Manipur, India \\ ${ }^{3}$ National Dairy Research Institute, Karnal Haryana 132001 \\ ${ }^{4}$ Department of Agronomy, UAS, Bangalore-560 065, Karnataka, India \\ ${ }^{5}$ Department Soil Science and Agricultural Chemistry, UAS, Bangalore, Karnataka, India \\ ${ }^{6}$ Deptartment of Soil Science and Agricultural Chemistry, Sericulture College, \\ Chintamani, Karnataka, India \\ *Corresponding author
}

\begin{tabular}{|c|c|}
\hline & A B S T R A C T \\
\hline & \multirow{7}{*}{ 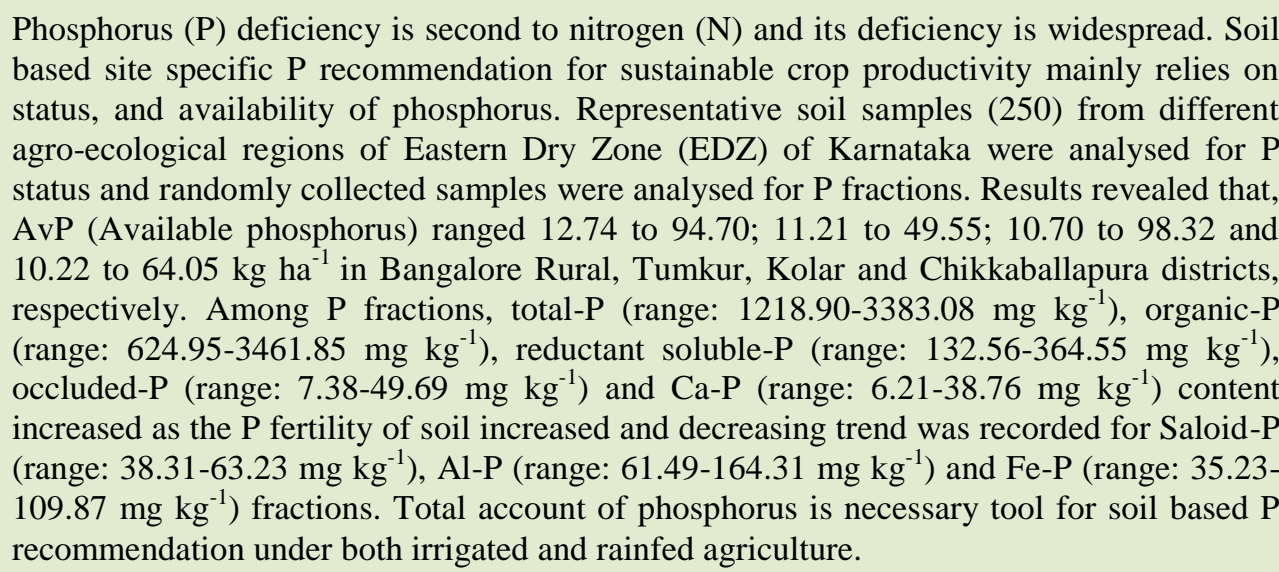 } \\
\hline & \\
\hline & \\
\hline $\begin{array}{l}\text { Dry zone Karnataka, } \mathrm{P} \\
\text { Fractions. }\end{array}$ & \\
\hline Article Info & \\
\hline $\begin{array}{l}\text { Accepted: } \\
\text { 04 September } 2017 \\
\text { Available Online: } \\
10 \text { November } 2017\end{array}$ & \\
\hline & \\
\hline
\end{tabular}

\section{Introduction}

Phosphorus has been the subject of intensive research because of its complex nature. The complexity arises because of three main factors. First, the total phosphorus level of soil is low. Second, the native phosphorus compounds are mostly unavailable for plant uptake, some being highly insoluble. Third, when soluble phosphorus sources such as those in manures and fertilizers are added to soil, they are readily transformed into unavailable forms and with time react further to become highly insoluble forms. Levels of different pools of soil $\mathrm{P}$ have been affected not only by soil properties and climatic condition but also by rate and type of $\mathrm{P}$ applied (Myungsu Park et al., 2006).

The total $\mathrm{P}$ content in agricultural crops generally ranges from $0.2-0.5$ per cent. Analysis of 3.65 million soil samples (1997 - 
1999) indicates that 42 per cent samples are low, 38 per cent medium and 20 per cent high in phosphorus (Motsara, 2002). There is an increasing pressure to reduce the application of fertilizers in commercial agriculture and minimize non-point sources of pollution of both surface and ground waters. There was a selective crop response to nutrients in different soils and the responsiveness varied with soil nutrient status (Mulla et al., 1992).

Continuous application of phosphorus results in buildup of this nutrient in the soil. The buildup of phosphorus depresses the availability of $\mathrm{Zn}$ and $\mathrm{S}$. However, when nutrient additions are less than the requirement, the crop draws the soil nutrients. With such continuous withdrawals, the native resources diminish with time. Therefore, application of soil based rather than uniform rates of fertilizers is must. Further, Import of DAP increased from 0.6 million tonnes to 2.7 million tonnes during 2007-2008. Thus, to realize maximum benefits and reduce nutrient losses from fertilizers, they must be applied in the right quantity and source based on initial soil nutrient status.

In the light of the above facts, soils of Easter Dry Zone of Karnataka were analysed for available phosphorus and $\mathrm{P}$ fractions with an objective is to assess the status of available phosphorus and different phosphorus fractions in soils with different fertility levels.

\section{Materials and Methods}

To know the available phosphorus status, 250 soil samples were collected from 4 AgroEcological Systems (AES) of Eastern Dry Zone of Karnataka (Fig. 1) covering parts of Tumkur, Bangalore (Urban and Rural areas), Chikkaballapura and Kolar districts.

The details of the sampling areas are presented in Table 1 along with $\mathrm{P}$ status.
Collected soil samples were air dried, powdered, passed through $2 \mathrm{~mm}$ sieve, stored in polythene bags and were analyzed for available phosphorus by adopting Jackson, 1973 procedure of Olsen's extraction method and Colorimetry for soils $\mathrm{pH}$ more than 6.5 and Brays extraction method for soils $\mathrm{pH}$ less than 6.5.

Based on the available phosphorus content, soils from EDZ of Karnataka were categorized as Very Low (VL: $<15 \mathrm{~kg} \mathrm{ha}^{-1}$ ), Low (L: 16-30 kg ha ${ }^{-1}$ ), Medium (M: 31-45 $\mathrm{kg} \mathrm{ha}{ }^{-1}$ ), High (H: 46-60 kg ha ${ }^{-1}$ ) and Very high (VH: > $60 \mathrm{~kg} \mathrm{ha}^{-1}$ ) categories. Three soils from each of these categories were selected randomly and analyzed for different $\mathrm{P}$ fractions using standard procedure as given in 2.1 .

\section{Forms of phosphorus}

\section{Total phosphorus}

The total phosphorus was extracted by digesting the soil with nitric acid and perchloric acid until a white residue was left. The residue was filtered and made to a known volume. Total phosphorus was then estimated by vanado-molybdo phosphoric yellow colour method (Hesse, 1971)

\section{Organic phosphorus}

Organic phosphorus was determined by deducting the sum of total inorganic phosphorus from total phosphorus as suggested by Mehta et al., (1954).

\section{Available phosphorus}

The available phosphorus was extracted using Bray's No.1 extractant for the soils having $\mathrm{pH}$ less than 6.5 and Olsen's extractant for the soils having $\mathrm{pH} 6.5$ and above. 
The extracted phosphorus was estimated by chloro-stannous reduced molybdo-phosphoric blue colour method (Jackson, 1973).

\section{Forms of inorganic phosphorus}

The method outlined by Peterson and Corey (1966) was followed to fractionate soil inorganic phosphorus.

\section{Saloid bound phosphorus (Saloid P)}

Exactly $0.5 \mathrm{~g}$ of soil was taken in to a $50 \mathrm{ml}$ polyethylene centrifuge tube, $25 \mathrm{ml}$ of $1 \mathrm{M}$ $\mathrm{NH}_{4} \mathrm{Cl}$ solution was added and shaken for 30 minutes. Saloid-P was estimated by molybdosulphuric acid reagent, using stannous chloride as reductant after taking the extract from supernatant solution after centrifugation in to an aliquot of $10 \mathrm{ml}$ isobutyl alcohol. Blue colour intensity was measured at $660 \mathrm{~nm}$ using spectrophotometer.

\section{Aluminium phosphorus (Al-P)}

The soil residue left after saloid-P estimation was shaken for one hour with $25 \mathrm{ml}$ of $0.5 \mathrm{M}$ $\mathrm{NH}_{4} \mathrm{~F}$ ( $\mathrm{pH}$ 8.2). The Al-P in the supernatant centrifuged suspension was determined by chloro-molybdic-boric acid reagent and chloro-stannous reductant. The intensity of blue colour developed was read in spectrophotometer at $660 \mathrm{~nm}$.

\section{Iron phosphorus (Fe-P)}

The soil sediment from Al-P estimation was washed twice with $25 \mathrm{ml}$ portion of saturated $\mathrm{NaCl}$ solution by shaking and centrifuging. The soil was then treated with $0.1 \mathrm{M} \mathrm{NaOH}$ and shaken for 17 hours and centrifuged. The supernatant solution was then treated with five drops of concentrated sulphuric acid. Phosphorus free activated carbon was used to remove suspended organic matter. The Fe-P content in the filtrate was determined by chloro-molybdic-boric acid reagent and chloro-stannous reductant. The intensity of blue colour developed was measured using spectrophotometer at $660 \mathrm{~nm}$.

\section{Reductant soluble phosphorus (R-P)}

The soil residue from $\mathrm{Fe}-\mathrm{P}$ estimation was washed twice with $25 \mathrm{ml}$ of saturated $\mathrm{NaCl}$ solution by shaking and centrifuging. Soil was then suspended in $15 \mathrm{ml}$ of $0.3 \mathrm{M}$ sodium citrate solution and shaken for 15 minutes with $0.5 \mathrm{~g}$ sodium dithionate. The suspension was heated on a water bath at $80{ }^{\circ} \mathrm{C}$ for a few minutes. Clear supernatant solution was decanted into a $50 \mathrm{ml}$ volumetric flask after centrifugation. Soil was then washed twice with saturated $\mathrm{NaCl}$ and the washings returned to sodium citrate-dithionate extract which was taken for R-P estimation. Excess of citrate and dithionate were oxidised by 1.5 $\mathrm{ml}$ of $0.25 \mathrm{M} \mathrm{KMnO}_{4}$ solution. The R-P was estimated by molybdate-sulphuric acid reagent with stannous chloride as reductant after taking the extract into an aliquot of 10 $\mathrm{ml}$ isobutyl alcohol. The blue colour intensity developed was diluted with equal quantity of absolute ethyl alcohol and read at $660 \mathrm{~nm}$ in spectrophotometer.

\section{Occluded phosphorus (Occl-P)}

The soil residue left out in the estimation of R-P was added with $25 \mathrm{ml}$ of $0.1 \mathrm{M} \mathrm{NaOH}$ and shaken for one hour. Supernatant solution after centrifugation was taken for estimation of Occl-P by chloro-molybdic-boric acid reagent with chloro-stannous reductant.

\section{Calcium phosphorus (Ca-P)}

The soil residue after extraction of occluded phosphorus was washed twice with $25 \mathrm{ml}$ of saturated $\mathrm{NaCl}$ solution and washings were discarded. Ca-P was extracted by using 0.25 $\mathrm{M} \mathrm{H}_{2} \mathrm{SO}_{4}$ and shaking for one hour and centrifuging for five minutes. The phosphorus in supernatant solution was estimated by 
chloro-molybdic-boric acid reagent with chloro-stannous reductant.

\section{Results and Discussion}

\section{P status}

The nature and distribution of different forms of $\mathrm{P}$ provides useful information for assessing the available $\mathrm{P}$ status of soil. Estimation of available $\mathrm{P}$ indicates only the amount of $\mathrm{P}$ present in soil solution and soil surface which is available to plants but it does not indicate about the relative contribution of different fractions of $\mathrm{P}$ towards available $\mathrm{P}$ (Lungmuana et al., 2012).

In Bangalore rural district, available phosphorus content ranged from 12.74 to $94.70 \mathrm{~kg} \mathrm{ha}^{-1}$ whereas in Tumkur district, it ranged from 11.21 to $49.55 \mathrm{~kg} \mathrm{ha}^{-1}$. Similarly in Kolar distict, the available phosphorus content of soil ranged from 10.70 to $98.32 \mathrm{~kg}$ $\mathrm{ha}^{-1}$ and it ranged from 10.22 to $64.05 \mathrm{~kg} \mathrm{ha}^{-1}$ in Chikkaballapura district (Table 1). The higher available $\mathrm{P}$ in soil may be due to buildup of $\mathrm{P}$ due to continuous addition of $\mathrm{P}$ fertilizer for the crops. Low in available $\mathrm{P}$ content of soil recorded may be due to regular cultivation with inadequate supply of phosphorus to crops. Higher levels of fertilizer $\mathrm{P}$ are needed in soils testing very low and low. Fertilizer P to be applied can be reduced when soils test very high in available P. Sharma et al., (2012) reported the available $P$ in Trans-Gangetic Plains, Upper Gangetic Plains, Middle Gangetic Plains and Lower Gangetic Plains was in the range of 6.7-85.1, $4.5-155.0$ and $4.7-183.7,2.2-112.0 \mathrm{~kg} \mathrm{ha}^{-1}$, respectively. Gurinderbir Singh and Sharma (2007) reported that the soils of Punjab showed low to high in available P.

Laxminarayana (2007) noticed Brays'1 available $\mathrm{P}$ status ranged from 6.56 to 10.93 $\mathrm{kg} \mathrm{P} \mathrm{ha}{ }^{-1}$ in rice growing soils of Mizoram.
Hasan (1996) reported that the available phosphorus status in Karnataka was ranged from low (16\%) to medium (3\%). Myungsu Park et al., (2006) reported that the higher level of $\mathrm{P}$ remaining in the soil is accumulated by long-term annual application of compost and chemical fertilizers than by that of chemical fertilizer, and $\mathrm{P}$ accumulation might be a gradual saturation of the P-sorption capacity.

\section{Categorization of soil available phosphorus $\left(\mathbf{P}_{2} \mathbf{O}_{5}\right)$}

Categorization of soil available phosphorus (Table 2) found that 43.20 per cent of soil samples comes under low $\left(<22.90 \mathrm{~kg} \mathrm{ha}^{-1}\right)$ and 43.20 per cent of soil samples comes under medium (22.9-56.33 kg ha' ${ }^{-1}$ ) category, which represents 108 samples each, in the total 250 samples. High (> $56.33 \mathrm{~kg} \mathrm{ha}^{-1}$ ) category showed 13.60 per cent (34 soil samples). Percentage of soil samples under different category are arranged in the ascending order as follows:

Low $=$ Medium $>$ High

\section{Phosphorus fractions}

$\mathrm{P}$ fractions in soils of different phosphorus status were determined by selecting three soil samples from each tentatively classified categories viz., very low $\left(<15 \mathrm{~kg} \mathrm{P}_{2} \mathrm{O}_{5} \mathrm{ha}^{-1}\right)$, low (16-30 $\mathrm{kg} \mathrm{P}_{2} \mathrm{O}_{5} \mathrm{ha}^{-1}$ ), medium (31-45 kg $\mathrm{P}_{2} \mathrm{O}_{5}$ ha $\left.^{-1}\right)$, high (46-60 $\mathrm{kg} \mathrm{P}_{2} \mathrm{O}_{5}$ ha $\left.^{-1}\right)$ and very high (> $60 \mathrm{~kg} \mathrm{P}_{2} \mathrm{O}_{5}$ ha $^{-1}$ ) categories (Table 2).

The T-P, Org-P, RS-P, Occl-P and Ca-P content of soil increased as the phosphorus fertility status of soil increased from very low to very high (Fig. 2). Mean values of total-P ranged from 1218.90 to $3383.08 \mathrm{mg} \mathrm{kg}^{-1}$. However, it ranged from 1082.09 to 1380.60 , $1641.79-2089.55,2164.18$ - 2201.49, and $2761.19-4067.16 \mathrm{mg} \mathrm{kg}^{-1}$ in very low, low, 
medium, high and very high $\mathrm{P}$ fertility soils, respectively.

The mean organic-P values was lower (709.33 $\mathrm{mg} \mathrm{kg}^{-1}$ ) in very low $\mathrm{P}$ fertility soil and was higher $\left(2819.10 \mathrm{mg} \mathrm{kg}^{-1}\right)$ in very high fertility soil. The values ranged $624.95-833.75$, 1204.06 - $1500.75,1664.60-1679.93$, $1970.06-2210.73$ and $2231.52-3461.85 \mathrm{mg}$ $\mathrm{kg}^{-1}$ in very low, low, medium, high and very high $\mathrm{P}$ fertility soils, respectively.

Table.1 Soil available phosphorus status in EDZ of Karnataka

\begin{tabular}{|c|c|c|c|}
\hline $\begin{array}{l}\text { Sl. } \\
\text { No. }\end{array}$ & $\begin{array}{c}\text { Agro-Ecological Situations (Name of the } \\
\text { taluk and village) }\end{array}$ & $\begin{array}{c}\text { Available } \mathrm{P}_{2} \mathrm{O}_{5} \\
\left(\mathrm{~kg} \mathrm{ha}^{-1}\right)\end{array}$ & $\begin{array}{l}\text { Previous crop } \\
\text { grown }\end{array}$ \\
\hline \multicolumn{4}{|c|}{ Bengaluru Rural District: Doddaballapurataluk } \\
\hline 1 & Saslu & 65.59 & Maize \\
\hline 2 & Saslu & 24.50 & Ragi \\
\hline 3 & Thadalabandde & 70.97 & Ragi \\
\hline 4 & Kankenahalli & 61.63 & Maize \\
\hline 5 & Adakavala & 26.37 & Maize \\
\hline 6 & Kadathippuru & 84.81 & Maize \\
\hline 7 & Akkatammanahalli & 16.37 & Ragi \\
\hline 8 & kattivasanahalli & 32.74 & Ragi \\
\hline 9 & Doddabelavangala & 31.20 & Ragi \\
\hline 10 & Sonnenahalli & 69.10 & Ragi \\
\hline 11 & Turuvanahally,Tubgere & 36.58 & Ragi \\
\hline 12 & Lakkasandra, Tubgere $(\mathrm{H})$ & 29.88 & Maize \\
\hline 13 & Tubgere $(\mathrm{H})$ & 67.45 & Maize \\
\hline 14 & Tubgere(H) & 79.98 & Maize \\
\hline 15 & Hadonahally & 58.12 & Maize \\
\hline 16 & Kansavadi & 30.32 & Ragi \\
\hline 17 & Honnavara & 18.90 & Ragi \\
\hline 18 & Purushanahally & 13.73 & Ragi \\
\hline 19 & Hambalgere & 18.13 & Ragi \\
\hline \multicolumn{4}{|c|}{ Bengaluru Rural District: Nelamangala taluk } \\
\hline 20 & Hegunda & 72.51 & Ragi \\
\hline 21 & Narasipura & 39.66 & Ragi \\
\hline 22 & Bugudihally & 29.77 & Ragi \\
\hline 23 & Makenahally & 51.19 & Ragi \\
\hline 24 & Enchenahally & 27.47 & Ragi \\
\hline 25 & Kundanahally & 39.88 & Ragi \\
\hline 26 & Adivasahalli, Thyamagondadlu & 16.15 & Ragi \\
\hline 27 & Thyamagondadlu & 29.55 & Ragi \\
\hline 28 & Thyamagondadlu & 13.29 & Ragi \\
\hline 29 & Thyamagondadlu & 23.29 & Ragi \\
\hline 30 & Kalghatta & 19.34 & Red gram \\
\hline 31 & Mallumghatteri & 49.99 & Ragi \\
\hline 32 & Thippaganahalli & 19.01 & Ragi \\
\hline 33 & Mallarabanavadi & 26.59 & Ragi \\
\hline 34 & Basavanahalli & 28.12 & Ragi \\
\hline 35 & Mylayahalli & 27.90 & Ragi \\
\hline 36 & Rampura & 69.32 & Potato \\
\hline
\end{tabular}




\begin{tabular}{|c|c|c|c|}
\hline 37 & Tindlu, & 51.85 & Red gram \\
\hline 38 & Neraganahally & 83.38 & Ragi \\
\hline 39 & Open filed Jail, Koramanagla & 35.70 & Ragi \\
\hline 40 & Open filed Jail, Koramanagla & 41.97 & Vegetables \\
\hline 41 & Open filed Jail,Koramanagla & 54.05 & Ragi \\
\hline 42 & Open filed Jail,Koramanagla & 17.91 & Maize \\
\hline 43 & Ahuti & 35.92 & Ragi \\
\hline 44 & Vijayapura & 48.56 & Ragi \\
\hline 45 & Vijayapura & 12.74 & Ragi \\
\hline 46 & Patna & 15.93 & Ragi \\
\hline 47 & Patna & 26.92 & Ragi \\
\hline 48 & Patna $(\mathrm{H})$ & 29.77 & Ragi \\
\hline 49 & Sulibele & 23.62 & Ragi \\
\hline 50 & Sulibele & 34.83 & Ragi \\
\hline 51 & Teneuoor, Sulibele & 30.87 & Ragi \\
\hline \multicolumn{4}{|c|}{ Bengaluru Rural District: Hoskotetaluk } \\
\hline 52 & Chikkaallalagere & 38.34 & Ragi \\
\hline 53 & Tharibehalli & 16.48 & Ragi \\
\hline 54 & Kariberanahosahalli & 94.70 & Ragi \\
\hline 55 & Kariberanahosahalli & 31.75 & Ragi \\
\hline 56 & Kariberanahosahalli & 13.62 & Ragi \\
\hline 57 & Hraluru, Haralemakanahalli & 31.31 & Ragi \\
\hline 58 & Chimundanahalli & 62.73 & Ragi \\
\hline 59 & Chimundanahalli & 87.78 & Ragi \\
\hline 60 & Kannuralli & 38.89 & Ragi \\
\hline 61 & Halapanahalli & 62.95 & Ragi \\
\hline 62 & Lakondahalli & 49.44 & Ragi \\
\hline 63 & Nandugudi & 40.87 & Ragi \\
\hline 64 & Banahalli & 25.71 & Ragi \\
\hline 65 & Indiganala & 20.65 & Ragi \\
\hline 66 & VaddarahalliTq & 22.85 & Ragi \\
\hline 67 & Araluru $\mathrm{Tq}$ & 49.11 & Ragi \\
\hline \multicolumn{4}{|c|}{ Tumkur district : Gubbitaluk } \\
\hline 68 & M.H. Patna, & 51.85 & Paddy \\
\hline 69 & Ammanaghatta & 32.30 & Ragi \\
\hline 70 & Channashettyhalli & 13.95 & Paddy \\
\hline 71 & Gubbi & 12.53 & Paddy \\
\hline 72 & Mattighata & 13.51 & Ragi \\
\hline 73 & Nittur & 11.21 & Ragi \\
\hline 74 & Kundernally & 19.45 & Ragi \\
\hline 75 & Kundernally & 14.39 & Ragi \\
\hline 76 & Doddaguni & 17.58 & Red gram \\
\hline 77 & Doddaguni & 15.60 & Red gram \\
\hline 78 & Godekeregate & 14.39 & Red gram \\
\hline \multicolumn{4}{|c|}{ Tumkur district : Chikkanayakanahallitaluk } \\
\hline 79 & Godekere Gate & 11.54 & Ragi \\
\hline 80 & Chikkanayakanahalli & 19.56 & Ragi \\
\hline 81 & Chikkanayakanahalli & 19.34 & Ragi \\
\hline 82 & Maligehalli & 17.36 & Ragi \\
\hline 83 & Balkere & 23.73 & Red gram \\
\hline
\end{tabular}




\begin{tabular}{|c|c|c|c|}
\hline 84 & Sulakatte & 17.69 & Ragi \\
\hline 85 & Sulakatte & 21.09 & Red gram \\
\hline \multicolumn{4}{|c|}{ Tumkur district : Tiptur } \\
\hline 86 & Mallenahally & 18.68 & Ragi \\
\hline 87 & Tiptur & 17.91 & Ragi \\
\hline 88 & Mattihally & 18.68 & Ragi \\
\hline 89 & Shankrikoppalu & 13.40 & Red gram \\
\hline 90 & Linganahally & 15.38 & Ragi \\
\hline 91 & B.G Palya & 18.79 & Ragi \\
\hline 92 & Sorekunte & 13.73 & Red gram \\
\hline 93 & Sorekunte & 49.55 & Ragi \\
\hline 94 & Dodderi & 16.04 & Ragi \\
\hline 95 & Ballapura & 20.43 & Ragi \\
\hline \multicolumn{4}{|c|}{ Kolar district } \\
\hline 96 & Jodikrishnapura & 28.89 & Ragi \\
\hline 97 & Achatnalli & 65.48 & Ragi \\
\hline 98 & Kurkinarasapura & 29.55 & Ragi \\
\hline 99 & Chowdenahalli & 30.21 & Ragi \\
\hline 100 & Karinakanahalli, Malur & 20.98 & Ragi \\
\hline 101 & Malur & 98.32 & Ragi \\
\hline 102 & Malur & 59.76 & Ragi \\
\hline 103 & Malur & 82.72 & Ragi \\
\hline 104 & Malleshwarnagar & 33.40 & Ragi \\
\hline 105 & Vakkaleri & 89.54 & Cauliflower \\
\hline 106 & Chinnapura & 57.57 & Ragi \\
\hline 107 & Dandiganahalli & 101.40 & Ragi \\
\hline 108 & Beglihosahalli & 24.72 & Ragi \\
\hline 109 & Ammerehally & 30.76 & Ragi \\
\hline 110 & Beglibenjanahalli & 33.40 & Ragi \\
\hline 111 & Chatrakodihally & 61.52 & Ragi \\
\hline 112 & Mudiyalla & 38.78 & Ragi \\
\hline 113 & Chatrakodihally & 61.52 & Ragi \\
\hline 114 & Mudiyalla & 38.78 & Ragi \\
\hline 115 & Vemgal & 28.45 & Ragi \\
\hline 116 & Kurngal & 68.33 & Ragi \\
\hline 117 & Harjenahally & 64.82 & Ragi \\
\hline 118 & Nagunalu & 57.13 & Ragi \\
\hline 119 & Nagunalu & 44.16 & Ragi \\
\hline 120 & Busunahalli & 53.39 & Ragi \\
\hline 121 & Busunahalli & 97.01 & Maize \\
\hline 122 & Hurugali & 58.99 & Ragi \\
\hline 123 & Oluru & 6.59 & Ragi \\
\hline 124 & Marenahalli & 35.92 & Ragi \\
\hline \multicolumn{4}{|c|}{ Kolardistrict:Mulabaglu } \\
\hline 125 & Mudiyannur & 48.01 & Ragi \\
\hline 126 & Kurudumalai & 48.67 & Ragi \\
\hline 127 & Kadaripura & 49.33 & Ragi \\
\hline 128 & Mulabaglu & 69.76 & Ragi \\
\hline 129 & Kuruibarahally & 60.20 & Ragi \\
\hline 130 & Nanagali & 33.62 & Ragi \\
\hline
\end{tabular}




\begin{tabular}{|c|c|c|c|}
\hline 131 & Patrahalli & 31.86 & Groundnut \\
\hline 132 & Pattarahally & 19.12 & Groundnut \\
\hline 133 & Gummakal & 36.25 & - \\
\hline 134 & Varadaganahalli & 48.69 & - \\
\hline 135 & Varadaganahalli & 32.96 & - \\
\hline 136 & Avani & 32.63 & - \\
\hline \multicolumn{4}{|c|}{ Kolardistrict:Bangarpet } \\
\hline 137 & Chikkankandahalli & 45.13 & Ragi \\
\hline 138 & Kannimbelle & 40.21 & Ragi \\
\hline 139 & Kannimbelle & 70.75 & Ragi \\
\hline 140 & Baydbelle & 22.85 & Ragi \\
\hline 141 & Nagashettahalli & 30.32 & Ragi \\
\hline 142 & Nagulahally & 46.91 & Ragi \\
\hline 143 & Kaysamballi & 27.03 & Ragi \\
\hline 144 & Kaysamballi & 21.97 & Ragi \\
\hline 145 & Lakshmisagar & 33.51 & Ragi \\
\hline 146 & Badgurki & 37.46 & Ragi \\
\hline 147 & Lekanahally & 24.39 & Ragi \\
\hline 148 & Chikkayelasandra & 27.25 & Ragi \\
\hline 149 & Dinakottur & 29.88 & Ragi \\
\hline \multicolumn{4}{|c|}{ Kolar district: Malur } \\
\hline 150 & Jagadenahalli, & 50.87 & Ragi \\
\hline 151 & Jagadenahalli & 29.55 & Ragi \\
\hline 152 & Thalikunte & 46.36 & Ragi \\
\hline 153 & Thirumalahatti & 37.57 & Ragi \\
\hline 154 & Dinnerihorohaali & 51.19 & Ragi \\
\hline 155 & Dinnerihorohaali & 34.61 & Ragi \\
\hline 156 & Allahalli & 51.63 & Ragi \\
\hline 157 & Banahalli & 53.83 & Ragi \\
\hline 158 & Chikkanayakanahali & 40.87 & Ragi \\
\hline 159 & Chikkanayakanahali & 39.33 & Ragi \\
\hline 160 & Chikkanayakanahali & 47.02 & Groundnut \\
\hline 161 & Chikkanayakanahali & 33.62 & Ragi \\
\hline 162 & Malur & 73.74 & Ragi \\
\hline \multicolumn{4}{|c|}{ Kolardistrict:Srinivasapura } \\
\hline 163 & Kappalli & 43.50 & Ragi \\
\hline 164 & Kondamari & 19.60 & Ragi \\
\hline 165 & Arganapalli & 22.40 & Ragi \\
\hline 166 & Kurukongpalli & 15.40 & - \\
\hline 167 & Bapapalli & 13.20 & Ragi \\
\hline 168 & Bhimapguntapalli & 15.00 & Groundnut \\
\hline 169 & Kempareddigarapalli & 12.80 & Ragi \\
\hline 170 & Yerramarapalli & 13.10 & Ragi \\
\hline 171 & Gollapalli & 19.90 & Tomato \\
\hline 172 & Manchanaikakote & 20.50 & Ragi \\
\hline 173 & Nalavanki & 14.00 & Ragi \\
\hline 174 & Jodikottapalli & 19.10 & Ragi \\
\hline 175 & Kasandra & 26.70 & Ragi \\
\hline 176 & Kadirolaladdy & 19.70 & Ragi \\
\hline 177 & Yelladdur & 18.40 & Ragi \\
\hline
\end{tabular}




\begin{tabular}{|c|c|c|c|}
\hline 178 & Kuruppalli & 13.70 & Ragi \\
\hline 179 & Kottapalli & 14.30 & Ragi \\
\hline 180 & Muthagalli & 43.90 & Ragi \\
\hline 181 & Neernalli & 28.10 & Ragi \\
\hline 182 & Srinivasapura & 22.80 & Ragi \\
\hline 183 & Champalli & 10.70 & Ragi \\
\hline 184 & Champalli & 19.10 & Ragi \\
\hline 185 & Doddapalli & 23.80 & Ragi \\
\hline 186 & Nittur & 11.70 & Ragi \\
\hline 187 & Kummagunta & 17.60 & Ragi \\
\hline \multicolumn{4}{|c|}{ Chikkaballapura district: } \\
\hline 188 & Muddenahally & 11.32 & Ragi \\
\hline 189 & Arur & 14.28 & Ragi \\
\hline 190 & Arur & 19.12 & Ragi \\
\hline 191 & Hurinavarahalli & 12.85 & Ragi \\
\hline 192 & Hurinavarahalli & 23.95 & Onion \\
\hline 193 & Chikkanagarahalli & 27.79 & Potato \\
\hline 194 & Mandikal & 64.05 & Beans \\
\hline 195 & Gudimadlikallu & 30.21 & Popcorn \\
\hline 196 & Mandikallu & 10.88 & Popcorn \\
\hline 197 & Mandikallu & 34.94 & Popcorn \\
\hline 198 & DesandraHosahally & 19.34 & Maize \\
\hline 199 & Marsnalli & 42.08 & Ragi \\
\hline 200 & Bungupe & 10.22 & Ragi \\
\hline 201 & Kolaranahally & 15.60 & Ragi \\
\hline \multicolumn{4}{|c|}{ Chikkaballapuradistrict:Gauribidanur } \\
\hline 202 & Bandaralahalli & 13.95 & Maize \\
\hline 203 & Basavapura & 12.96 & Maize \\
\hline 204 & Bencchikkanahalli & 34.61 & Maize \\
\hline 205 & Begalkapura & 36.91 & Maize \\
\hline 206 & T.Bommasandra & 55.04 & Maize \\
\hline 207 & Vodalveni & 25.16 & Maize \\
\hline 208 & Demgattanahally & 15.82 & Maize \\
\hline 209 & Gulur & 31.37 & Sunflower \\
\hline \multicolumn{4}{|c|}{ Chikkaballapuradistrict:Gudibande } \\
\hline 210 & Gudibande & 11.80 & Maize \\
\hline 211 & Kondireddypalli & 31.50 & Ragi \\
\hline 212 & Adinarayanahally, & 11.60 & Ragi \\
\hline 213 & Chenduru & 15.30 & Ragi \\
\hline 214 & Bichaganahally & 43.90 & - \\
\hline 215 & Yarlekkanahally & 14.10 & Ragi \\
\hline 216 & Chinnappanahally & 15.10 & Groundnut \\
\hline 217 & Katenahally & 16.80 & Ragi \\
\hline \multicolumn{4}{|c|}{ Chikkaballapuradistrict:Baagepalli } \\
\hline 218 & Pottavarahally, & 18.50 & Ragi \\
\hline 219 & Mittemari & 14.70 & Red gram \\
\hline 220 & Burgamadgu & 14.20 & Red gram \\
\hline 221 & Gollapalli & 14.90 & Bhendi \\
\hline 222 & Achepalli & 14.50 & Red gram \\
\hline 223 & Guttamindapalli & 15.30 & Groundnut \\
\hline
\end{tabular}




\begin{tabular}{|c|l|l|l|}
\hline 224 & Sankatpalli & 28.70 & Maize \\
\hline 225 & Sigalapalli & 16.30 & Groundnut \\
\hline \multicolumn{2}{|l|}{ Chikkaballapuradistrict:Chintamani } & 43.50 & Ragi \\
\hline 226 & Hirepalya & 19.60 & Ragi \\
\hline 227 & Gunnahalli & 22.40 & Ragi \\
\hline 228 & Madabahalli & 15.40 & Ragi \\
\hline 229 & Perumachanahalli & 13.20 & Ragi \\
\hline 230 & Karatahalli & 15.00 & Ragi \\
\hline 231 & Kachahalli & 12.80 & Ragi \\
\hline 232 & Kuruburu & 13.10 & Potato \\
\hline 233 & Kuruburu & 19.90 & Potato \\
\hline 234 & Kuruburu & 20.50 & Ragi \\
\hline 235 & Bachivaralahalli & 14.00 & Ragi \\
\hline 236 & D.K.Halli & 19.10 & Ragi \\
\hline 237 & Chikkakarakamakalahalli & 26.70 & RAgi \\
\hline 238 & Karakamakanahalli & 19.70 & Ragi \\
\hline 239 & Eraganapalli & 18.40 & Tomato \\
\hline 240 & Erganapalli & 22.80 & Ragi \\
\hline 241 & Raipalli & 10.70 & Ragi \\
\hline 242 & Chintamani & 19.10 & Ragi \\
\hline 243 & Mohammedpura & 11.70 & Ragi \\
\hline 244 & Chikkatekahalli & & \\
\hline Chikkaballapuradistrict:Siddlaghatta & 13.70 & Ragi \\
\hline 245 & Andignala, & 14.30 & - \\
\hline 246 & Bhudala & 43.90 & Ragi \\
\hline 247 & Bachahalli & 28.10 & Ragi \\
\hline 248 & Kadranayakanahalli & 23.80 & Ragi \\
\hline 249 & Doddatekahalli & 17.60 & Ragi \\
\hline 250 & Kachanayakanahally & \\
\hline
\end{tabular}

Table.2 Categorization of available soil phosphorus $\left(\mathrm{P}_{2} \mathrm{O}_{5}\right)$ in Eastern Dry Zone of Karnataka

\begin{tabular}{|c|c|c|c|c|}
\hline \multirow{2}{*}{$\begin{array}{c}\text { AES/ } \\
\text { Phosphorus fertility status }\end{array}$} & $\begin{array}{c}\text { Cow } \\
(<\mathbf{2 2 . 9} \\
\left.\mathbf{k g ~ h a}^{-\mathbf{1}}\right)\end{array}$ & $\begin{array}{c}\text { Medium } \\
(\mathbf{2 2 . 9}-\mathbf{5 6 . 3 3} \\
\left.\mathbf{k g ~ h a}^{\mathbf{- 1}}\right)\end{array}$ & $\begin{array}{c}\text { High } \\
(>\mathbf{5 6 . 3 3} \\
\left.\mathbf{k g ~ h a}^{-\mathbf{1}}\right)\end{array}$ & $\begin{array}{c}\text { Total number of } \\
\text { samples }\end{array}$ \\
\hline Bangalore rural & 15 & 37 & 15 & 67 \\
\hline Tumkur & 24 & 4 & - & 28 \\
\hline Kolar & 25 & 49 & 18 & 92 \\
\hline Chikkaballapura & 44 & 18 & 1 & 63 \\
\hline Total number of samples & 108 & 108 & 34 & $\mathbf{2 5 0}$ \\
\hline Percentage of samples & $\mathbf{4 3 . 2}$ & $\mathbf{4 3 . 2}$ & $\mathbf{1 3 . 6}$ & \\
\hline
\end{tabular}


Table.3 Status of phosphorus fractions $\left(\mathrm{mg} \mathrm{kg}^{-1}\right)$ in different phosphorus fertility soils of EDZ of Karnataka

\begin{tabular}{|c|c|c|c|c|c|c|c|c|}
\hline P levels/ Soils & Total- $\mathbf{P}$ & Organic- P & $\mathbf{S}-\mathbf{P}$ & Al-P & Fe-P & RS-P & Occluded- P & Ca-P \\
\hline \multicolumn{9}{|c|}{ 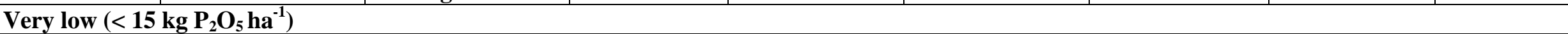 } \\
\hline Soil 1 & 1380.60 & 833.75 & 63.23 & 164.31 & 109.87 & 188.49 & 12.30 & 8.65 \\
\hline Soil 2 & 1194.03 & 669.32 & 55.54 & 163.81 & 106.80 & 180.20 & 10.82 & 7.54 \\
\hline Soil 3 & 1082.09 & 624.95 & 54.99 & 150.04 & 105.96 & 132.56 & 7.38 & 6.21 \\
\hline Mean & 1218.90 & 709.33 & 57.92 & 159.39 & $\mathbf{1 0 7 . 5 4}$ & 167.08 & 10.17 & 7.47 \\
\hline Range & 1082.09 -1380.60 & 624.95-833.75 & 54.99-63.23 & 150.04-164.31 & 105.96-109.87 & 132.56-188.49 & 7.38-12.30 & 6.21-8.65 \\
\hline \multicolumn{9}{|c|}{ Low $\left(15-30 \mathrm{~kg} \mathrm{P}_{2} \mathrm{O}_{5} \mathrm{ha}^{-1}\right)$} \\
\hline Soil 1 & 2089.55 & 1500.75 & 51.69 & 148.56 & 102.89 & 250.63 & 17.71 & 17.32 \\
\hline Soil 2 & 1828.36 & 1339.04 & 46.19 & 92.98 & 84.43 & 234.05 & 16.23 & 15.44 \\
\hline Soil 3 & 1641.79 & 1204.06 & 46.00 & 84.12 & 75.49 & 205.06 & 13.28 & 13.78 \\
\hline Mean & 1853.23 & 1347.96 & 47.96 & $\mathbf{1 0 8 . 5 5}$ & 87.60 & 229.91 & 15.74 & 15.51 \\
\hline Range & 1641.79-2089.55 & 1204.06-1500.75 & 46.00-51.69 & 84.12-148.56 & 75.49-102.89 & $205.06-250.63$ & 13.28-17.71 & 13.78-17.32 \\
\hline \multicolumn{9}{|c|}{ Medium (31-45 kg $\left.\mathrm{P}_{2} \mathrm{O}_{5} \mathrm{ha}^{-1}\right)$} \\
\hline Soil 1 & 2201.49 & 1678.30 & 46.00 & 83.14 & 74.37 & 273.41 & 21.65 & 24.62 \\
\hline Soil 3 & 2164.18 & 1679.93 & 43.80 & 78.22 & 67.38 & 256.84 & 18.20 & 19.81 \\
\hline Mean & 2176.61 & 1674.28 & 44.72 & 80.84 & 70.17 & 264.43 & 20.17 & 22.00 \\
\hline Range & 2164.18-2201.49 & $1664.60-1679.93$ & 43.80-46.00 & 78.22-83.14 & 67.38-74.37 & 256.84-273.41 & 18.20-21.65 & 19.81-24.62 \\
\hline \multicolumn{9}{|c|}{$\operatorname{High}\left(46-60 \mathrm{~kg} \mathrm{P}_{2} \mathrm{O}_{5} \mathrm{ha}^{-1}\right)$} \\
\hline Soil 1 & 2761.19 & 2207.38 & 43.80 & 77.73 & 63.74 & 298.26 & 39.85 & 30.43 \\
\hline Soil 2 & 2723.88 & 2210.73 & 42.52 & 76.25 & 47.81 & 287.91 & 30.01 & 28.65 \\
\hline Soil 3 & 2462.68 & 1970.06 & 41.61 & 72.31 & 42.78 & 285.84 & 23.61 & 26.47 \\
\hline Mean & 2649.25 & 2129.39 & 42.64 & 75.43 & 51.44 & 290.67 & 31.16 & 28.52 \\
\hline Range & 2462.68-2761.19 & 1970.06-2210.73 & 41.61-43.80 & 72.31-77.73 & 42.78-63.74 & 285.84-298.26 & 23.61-39.85 & 26.47-30.43 \\
\hline \multicolumn{9}{|c|}{ Very high $\left(>60 \mathrm{~kg} \mathrm{P}_{2} \mathrm{O}_{5} \mathrm{ha}^{-1}\right)$} \\
\hline Soil 1 & 4067.16 & 3461.85 & 41.42 & 72.31 & 38.58 & 364.55 & 49.69 & 38.76 \\
\hline Soil 2 & 3320.89 & 2763.95 & 40.87 & 71.82 & 36.62 & 325.19 & 48.70 & 33.74 \\
\hline Soil 3 & 2761.19 & 2231.52 & 38.31 & 61.49 & 35.23 & 316.91 & 45.75 & 31.98 \\
\hline Mean & 3383.08 & 2819.1 & 40.20 & 68.54 & 36.81 & 335.55 & 48.05 & 34.83 \\
\hline Range & 2761.19-4067.16 & 2231.52-3461.85 & 38.31-41.42 & 61.49-72.31 & 35.23-38.58 & 316.91-364.55 & 45.75-49.69 & 31.98-38.76 \\
\hline
\end{tabular}


Table.4 Per cent contribution of different forms of phosphorus to total phosphorus

\begin{tabular}{|l|l|l|l|l|l|l|l|}
\hline P levels & Org-P & S-P & Al-P & Fe-P & RS-P & Occl- P & Ca-P \\
\hline Very low & 58.19 & 4.75 & 13.07 & 8.82 & 13.70 & 0.84 & 0.61 \\
\hline Low & 72.74 & 2.58 & 5.86 & 4.73 & 12.41 & 0.85 & 0.84 \\
\hline Medium & 76.92 & 2.05 & 3.72 & 3.23 & 12.15 & 0.93 & 1.01 \\
\hline High & 80.37 & 1.61 & 2.85 & 1.94 & 10.97 & 1.17 & 1.07 \\
\hline Very high & 83.33 & 1.19 & 2.03 & 1.08 & 9.92 & 1.42 & 1.03 \\
\hline
\end{tabular}

Fig.1 Agro-climatic Zones of Karnataka indicating soil sampled area of Eastern Dry Zone

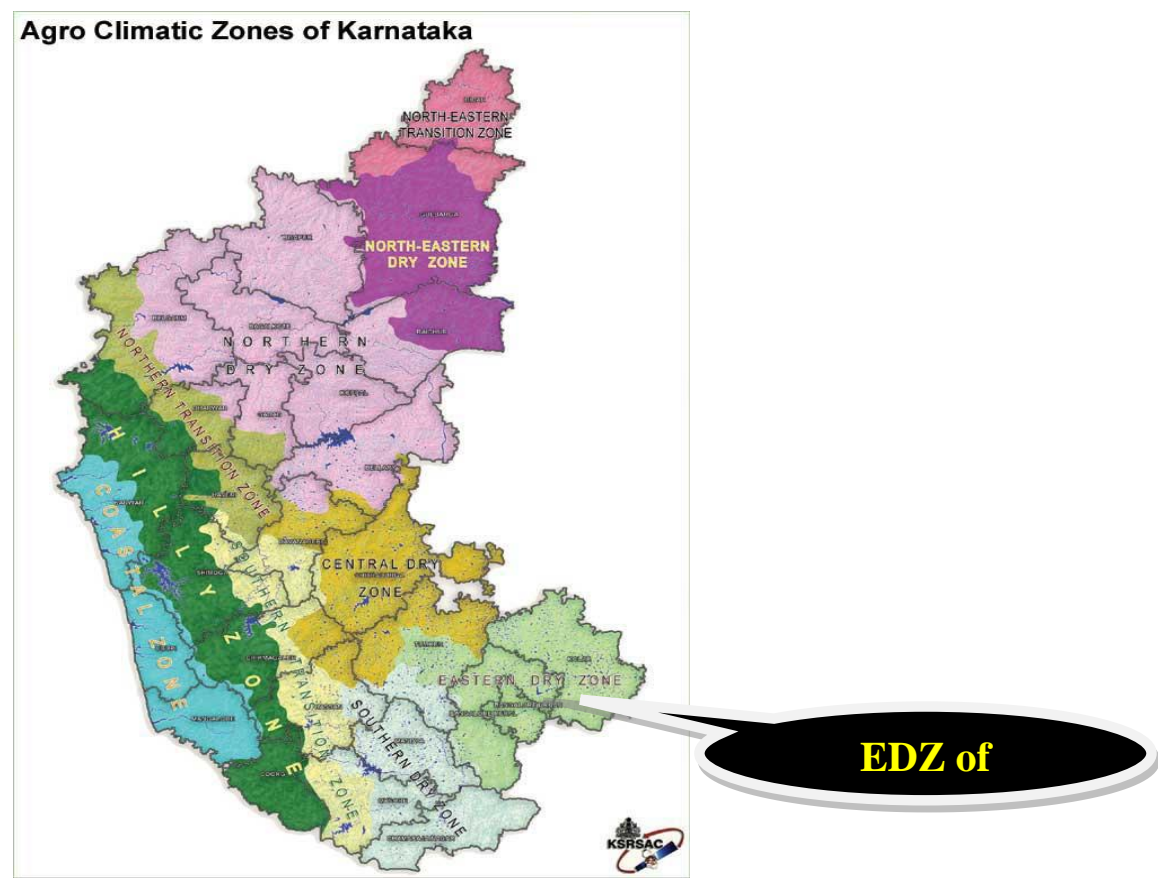

Fig.2 Categorization of soil available phosphorus content

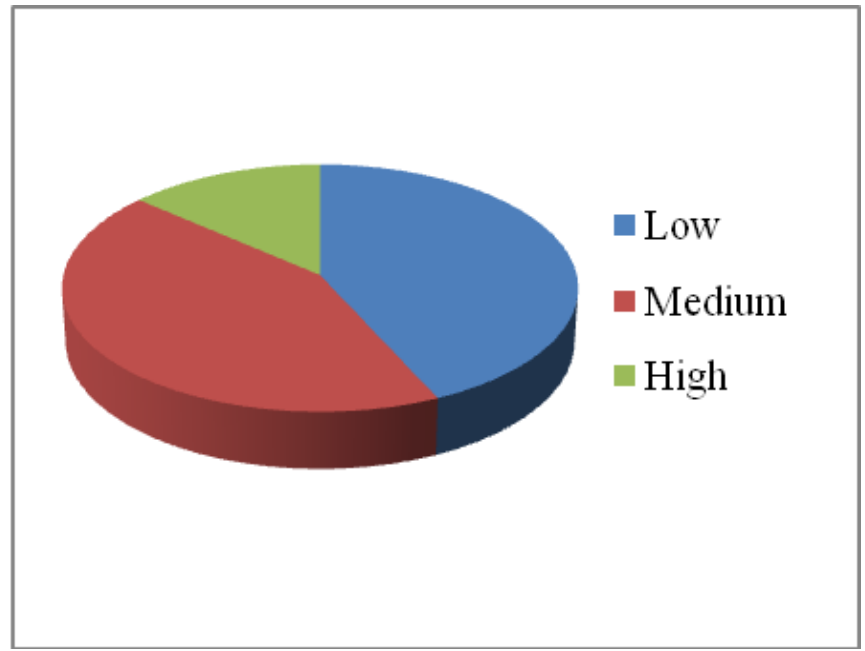


Similarly, RS-P ranged from 132.56 - 188.49 (mean of 167.08), $205.06-250.63$ (mean of 229.91), 256.84 - 273.41 (mean of 264.43), $285.84-298.26$ (mean of 290.67) and 316.91 - 364.55 (mean of 335.55 ) $\mathrm{mg} \mathrm{kg}^{-1}$, in very low, low, medium, high and very high $\mathrm{P}$ fertility soils, respectively.

Further, Occl-P in very low, low, medium, high and very high $\mathrm{P}$ fertility soils showed mean value of 10.17 (range 7.38 - 12.30), 15.74 (range 13.28 - 17.71), 20.17 (range 18.20 - 21.65), 31.16 (range 23.61 - 39.85) and 48.05 (range $45.75-49.69$ ) $\mathrm{mg} \mathrm{kg}^{-1}$, respectively. Ca-P in very low, low, medium, high and very high $\mathrm{P}$ fertility soils showed mean value of 7.47 (range $6.21-8.65$ ), 15.51 (range 13.78 - 17.32), 22.0 (range 19.81 24.62), 28.52 (range $26.47-30.43$ ) and 34.83 (range $31.98-38.76) \mathrm{mg} \mathrm{kg}^{-1}$, respectively.

Unlike T-P, Org-P, RS-P, Occl-P and Ca-P, $\mathrm{S}-\mathrm{P}, \mathrm{Al}-\mathrm{P}$ and $\mathrm{Fe}-\mathrm{P}$ recorded decreasing trend as the phosphorus fertility status of soil increased from very low to very high. S-P content of soil ranged from $54.99-63.23$ (mean of 57.92), 46.0 - 51.69 (mean of 47.96), 43.80 - 46.0 (mean of 44.72), 41.61 43.80 (mean of 42.64) and $38.31-41.42$ (mean of 40.20) $\mathrm{mg} \mathrm{kg}^{-1}$, in very low, low, medium, high and very high $\mathrm{P}$ fertility soils, respectively.

Al-P in very low, low, medium, high and very high $\mathrm{P}$ fertility soils showed mean value of 159.39 (range 150.04 - 164.31), 108.55 (range 84.12 - 148.56), 80.84 (range 78.22 83.14), 75.43 (range $72.31-77.73$ ) and 68.54 (range $61.49-72.31$ ) $\mathrm{mg} \mathrm{kg}^{-1}$, respectively. Similarly, Fe-P content of soil ranged from 105.96 - 109.87 (mean of 107.54), 75.49 102.89 (mean of 87.60), $67.38-74.37$ (mean of 70.17), $42.78-63.74$ (mean of 51.44) and 35.23 - 38.58 (mean of 36.81 ) $\mathrm{mg} \mathrm{kg}^{-1}$, in very low, low, medium, high and very high $\mathrm{P}$ fertility soils, respectively.
Total- $\mathrm{P}$, organic-P, reductant soluble-P, occluded-P and Ca-P content of soil increased as the phosphorus fertility of soil increased from very low to very high may be due to continuous addition of $\mathrm{P}$ sources to soil for the cultivation of crops which might have transformed the added $\mathrm{P}$ into these forms. The $\mathrm{S}-\mathrm{P}, \mathrm{Al}-\mathrm{P}$ and Fe-P recorded decreasing trend as the phosphorus fertility status of soil increased from very low to very high which may be due to transformation of these S-P, $\mathrm{Al}-\mathrm{P}$ and $\mathrm{Fe}-\mathrm{P}$ forms in to non-labile forms resulting in their lower availability. The sequential distribution of $\mathrm{P}$ fractions in different gradient strips followed the order;

Very low $\mathrm{P}$ fertility soils: Total-P $>$ Organic$\mathrm{P}>\mathrm{RS}-\mathrm{P}>\mathrm{Al}-\mathrm{P}>\mathrm{Fe}-\mathrm{P}>\mathrm{S}-\mathrm{P}>\mathrm{Occluded}-\mathrm{P}>\mathrm{Ca}-\mathrm{P}$

Low $\mathrm{P}$ fertility soils: Total-P $>$ Organic-P $>$ RS$\mathrm{P}>\mathrm{Al}-\mathrm{P}>\mathrm{Fe}-\mathrm{P}>\mathrm{S}-\mathrm{P}>$ Occluded-P $>\mathrm{Ca}-\mathrm{P}$

Medium $\mathrm{P}$ fertility soils: Total-P>Organic$\mathrm{P}>\mathrm{RS}-\mathrm{P}>\mathrm{Al}-\mathrm{P}>\mathrm{Fe}-\mathrm{P}>\mathrm{S}-\mathrm{P}>\mathrm{Ca}-\mathrm{P}>$ Occluded-P

High P fertility soils: Total-P $>$ Organic-P $>$ RS$\mathrm{P}>\mathrm{Al}-\mathrm{P}>\mathrm{Fe}-\mathrm{P}>\mathrm{S}-\mathrm{P}>$ Occluded-P $>\mathrm{Ca}-\mathrm{P}$

Very high $\mathrm{P}$ fertility soils: Total-P $>$ Organic$\mathrm{P}>\mathrm{RS}-\mathrm{P}>\mathrm{Al}-\mathrm{P}>\mathrm{Occluded}-\mathrm{P}>\mathrm{S}-\mathrm{P}>\mathrm{Fe}-\mathrm{P}>\mathrm{Ca}-\mathrm{P}$

Lungmuana et al., (2012) reported that on an average the percentage contribution of different fraction to the total $\mathrm{P}$ was in the order of RS-P $(28.2 \%)>$ Fe-P $(18.5 \%)>$ Al$\mathrm{P}(7.9 \%)>\mathrm{Ca}-\mathrm{P}(5.8 \%)>\mathrm{S}-\mathrm{P}(0.8 \%)$ in surface acidic soils of rice growing areas of red and Laterite zone of West Bengal. Laxminarayana (2007) reported that the total $\mathrm{P}$ in various rice growing soils of Mizoram ranged from 132.30 to $365.80 \mathrm{mg} \mathrm{kg}^{-1}$ with a mean of $242.50 \mathrm{mg} \mathrm{kg}^{-1}$. The sequential distribution of inorganic $\mathrm{P}$ fractions followed the order of RS-P (34.0 per cent) > Al-P $(19.60$ per cent $)>$ Fe-P $(15.80$ per cent $)>C$ Ca$\mathrm{P}$ (12.0 per cent) > S-P (2.46 per cent). The 
proportion of forms of phosphorus such as Ca-P, Al-P, Fe-P, reductant soluble-P, organic-P governs the response to applied $\mathrm{P}$ (Singh et al., 2003).

Percent contribution of different forms of phosphorus to total phosphorus (Table 3) showed that organic-P, occluded-P and calcium-P fractions contribution to T-P, increased with increase in $\mathrm{P}$ fertility of soils, whereas S-P, Al-P, Fe-P and RS-P showed reverse trend. Organic-P fraction contributed highest per cent (58.19 to 83.33 per cent in very low to very high fertility soils, respectively) followed by RS-P (9.92 to 13.70 per cent in very high to very low fertility soils, respectively) and $\mathrm{Ca}-\mathrm{P}$ contributed lower percent $(0.61$ to 1.03 percent in very low to very high fertility soils, respectively).

Available $\mathrm{P}_{2} \mathrm{O}_{5}$ in soils of EDZ of Karnataka ranged 10.22 to $98.32 \mathrm{~kg} \mathrm{ha}^{-1}$. Categorization of soil $\mathrm{P}$ recorded 43.20 per cent samples low and 43.20 per cent samples medium and 13.60 per cent samples belongs to high category. Total-P, organic-P, reductant soluble-P, occluded-P and Ca-P increased as the soil phosphorus fertility increased and reverse trend was noticed for saloid-P, Al-P and Fe-P fractions. Data on available $\mathrm{P}$ status and $\mathrm{P}$ fractions provide basis for soil specific $\mathrm{P}$ recommendation for sustainable $\mathrm{P}$ management both in irrigated and rainfed agriculture apart from reducing $\mathrm{P}$ losses and minimising pollution.

\section{Acknowledgments}

I would like to thank chairman and members of advisory committee, staff's of Dept. of SS\&AC, UAS, Bangalore, NBSS\&LUP and ICAR and my beloved family members and friends. Thankful to ICAR, New Delhi for granting SRF and RKVY, Govt. of Karnataka for providing financial assistance for conducting research.

\section{References}

Gurinderbir Singh and Sharma, (2007) Characterization of inorganic soil $\mathrm{P}$ forms in soils representing different agro-ecological zones of Punjab. $J$. Indian Soc. Soil Sci., 55(2), 209-211.

Hasan, R. (1996) Phosphorous status of soils in India. Better crops Int., 10(2), 4-5.

Hesse, P. R. (1971) A Text Book of Soil Chemical Analysis. John Murry (Publ) Ltd., London, U. K.

Jackson, M. L. (1973) Soil Chemical Analysis. Prentice Hall of India Private Limited, New Delhi, 485.

Laxminarayana, K. (2007) Distribution of inorganic $\mathrm{P}$ fractions and critical limits of available $\mathrm{P}$ in rice soils of Mizoram. J. Indian Soc. Soil Sci., 55(4), 481-487.

Lungmuana, Ghosh, S. K. and Patra, P. K. (2012) Distribution of different forms of phosphorus in surface soils of rice growing areas of red and laterite zone of West Bengal. J. Indian Soc. Soil Sci., 60(3), 204-207.

Mehta, N. C., Legg, J. O. and Black, C. A. (1954) Determination of organic phosphorus in soils: I. Extractions methods. Soil Sci. Soc. America. Proc., 18, 443-449.

Motsara, M. R. (2002) Available nitrogen, phosphorus and potassium status of Indian soils as depicted by soil fertility maps. Fert. News, 47(8), 15 - 21.

Mulla, D. J., Bhatti, A. U., Hammond, M. W. and Benson, J. A. (1992) A comparison of winter yield and quality under uniform versus spatially variable fertilizer management. Agric. Ecos. Envi, 38, 301-311.

Myungsu Park, Olayvanh Singvilay, Wansik Shin, Eunhee Kim, Jongbae Chung \& Tongmin Sa., (2006) Effects of Long-Term Compost and Fertilizer Application on Soil Phosphorus Status Under Paddy Cropping System. Comm. 
Soil Sci. and Plant Anal., 35(11-12), 1635-1644.

Peterson, G. W. and Corey, R. B. (1966) A modified Chang and Jackson procedure for routine fractionation of inorganic soil phosphates. Soil Sci. Soc. America Proc., 30, 563-565.

Sharma, B. D., Sidhu, G. S., Sarkar, D. and Kukal, S. S. (2012) Soil organic carbon, phosphorous, and potassium status in rice-wheat soils of different AgroClimatic Zones in Indo- Gangetic Plains of India. Comm. Soil Sci. Pl. Anal., 43, 1449-1467.

Singh, S. K., Baser, B. L., Shyampura, R. L. and Narain, P. (2003) Phosphorus fractions and their relationship to weathering indices in Vertisols. $J$. Indian Soc. Soil Sci., 51, 247-251.

\section{How to cite this article:}

Chandrakala, M., C.A. Srinivasamurthy, Sanjeev Kumar, S. Bhaskar, V.R.R. Parama and Naveen, D.V. 2017. Phosphorus Status in Soils of Eastern Dry Zone, Karnataka, India. Int.J.Curr.Microbiol.App.Sci. 6(11): 310-324. doi: https://doi.org/10.20546/ijcmas.2017.611.035 